of Treg compared with the pre-depletion group. Furthermore, the post-depletion group also exhibited lower fibrogenic cytokine-producing $T$ cell frequencies, suggesting that the change in T cell cytokine production could not account for the more strongly reduced fibrosis observed in the pre-depletion group. Therefore, we examined other immune cell response to B cell depletion. Recent studies have revealed that macrophages are divided into two subtypes: M1 and M2 and that M2 macrophages show fibrogenic effects in SSc. This study showed that macrophages cultured with B cells from BLM-induced SSc mice exhibited enhanced M2 differentiation compared with control B cells. Remarkably, frequencies of M2 macrophage with fibrogenic capacity significantly decreased in the pre-depletion group compared with the post-depletion group, which could account for the more strongly inhibited tissue fibrosis in the pre-depletion group.

Conclusions: Our results indicate that therapeutic effects of $B$ cell depletion on tissue fibrosis exert through regulating macrophage differentiation rather than $T$ cell cytokine production in SSc, first demonstrating that interaction between $\mathrm{B}$ cells and macrophages in development of fibrosis in SSc.

Disclosure of Interest: None declared

DOI: 10.1136/annrheumdis-2018-eular.4655

\section{FRI0413 ARYL HYDROCARBON RECEPTOR EXPRESSION IS ASSOCIATED WITH LUNG INVOLVEMENT IN SYSTEMIC SCLEROSIS}

H. Takei ${ }^{1}$, H. Yasuoka ${ }^{1}$, K. Yoshimoto ${ }^{1,2}$, K. Yamaoka ${ }^{1}$, T. Takeuchi ${ }^{1}{ }^{1}$ Division of Rheumatology, Department of Internal Medicine, Keio University School of Medicine; ${ }^{2}$ Clinical and Translational Research Center, Keio University Hospital, Tokyo, Japan

Background: Systemic sclerosis (SSc) is characterised by autoantibody production, microvascular injury and systemic excessive fibrosis ${ }^{1}$. Genetic and environmental factors are thought to be important for the trigger of development of the disease, however, direct connexion between these factors and pathogenesis of SSc is not yet elucidated ${ }^{23}$. Recent reports showed that environmental toxic pollutants, such as dioxins, play a significant role in the disturbance of immune system and the trigger of autoimmunity through binding aryl hydrocarbon receptor $(A h R)^{4) 5}$. However, little is known about the association between AhR and pathogenesis of SSc yet.

Objectives: To elucidate the association between AhR and the clinical characteristics of SSc.

Methods: Twenty-one patients with SSc who fulfilled 2013 ACR/EULAR classification criteria and 10 healthy controls $(\mathrm{HC})$ were analysed. Peripheral blood mononuclear cells (PBMCs) were isolated from heparinized whole blood by using gradient centrifugation and total RNA was prepared from PBMCs. Expression of Ahr mRNA was detected by quantitative polymerase chain reaction and standardised by mRNA level of $18 S$ ribosomal RNA in each sample. Level of Ahr mRNA in the cells was compared between SSc and HC and also between SSc patients with and without various clinical features.

Results: The proportion of diffuse cutaneous subset (dcSSc) was 33\%. Mean disease duration was $9 \pm 9$ years. The positive proportion of anticentromere antibodies, anti-topoisomerase I antibodies, anti-U1 ribonucleoprotein antibody, antiRNA polymerase III antibody and antinuclear antibody positive without SSc-specific antibodies among 21 patients were 33.3\%, 33.3\%, 4.8\%, 9.5\% and $9.5 \%$, respectively. Antinuclear antibody was negative in $2(9.5 \%)$ patients. Ahr mRNA expression level was tended to be higher in SSc compared to $\mathrm{HC}(1.7 \pm 1.1$ versus $1.2 \pm 0.6, p=0.1)$. Notably, the expression level of $A h R$ mRNA in dc SSc was tended to be higher than that of limited cutaneous SSc $(p=0.15)$, whereas no significant difference was detected between with or without SSc related autoantibodies, vasculopathy such as pulmonary arterial hypertension or digital tip ulcer. Importantly, the expression level of $A h r$ mRNA was significantly higher in patients with interstitial lung disease (ILD) $(n=14)$ than those without $(n=7)(2.0 \pm 1.1$ versus $1.0 \pm 0.4, \mathrm{p}<0.05)$. Furthermore, $A h R$ mRNA expression level was significantly and negatively correlated with DLCO $\%$ predicted $(r=-0.57, p<0.05)$.

Conclusions: Expression level of Ahr mRNA was higher in patients with SSc, especially in SSc patients with ILD. In addition, AhR expression level was correlated with a parameter of pulmonary function test, DLCO\% predicted. These results collectively suggest that AhR possibly plays an important role in the disease process of ILD in SSc.

\section{REFERENCES:}

[1] Nat Rev Rheumatol 2013;9(8):451-64.

[2] Nat. Rev. Rheumatol 2014;10:671-681.

[3] Semin. Immunopathol 2015;37:463-473.

[4] Annu Rev Immunol 2014;32:403-32.

[5] Nature. 2008;453(7191):65-71.

Disclosure of Interest: None declared

DOI: 10.1136/annrheumdis-2018-eular.6594

\section{FRI0414 \\ EVALUATION OF A NOVEL MULTI-ANALYTE ASSAY FOR THE DETECTION OF AUTOANTIBODIES IN THE DIAGNOSIS OF SYSTEMIC SCLEROSIS}

P.L. Meroni ${ }^{1}$, T. Schioppo ${ }^{2}$, F. Ingegnoli ${ }^{2,3}$, M.O. Borghi ${ }^{1,3}$, J.L. Milo ${ }^{4}$, C. Bentow ${ }^{4}$, A. Seaman ${ }^{4}$, G. Valentini ${ }^{5}$, S. Vettori $i^{5}$, P. Di Benedetto ${ }^{6}$, V. Liakouli ${ }^{6}$,

R. Giacomelli ${ }^{6}$, M. Mahler ${ }^{4} .{ }^{1}$ IImmunorheumatology Research Laboratory, Irccs Istituto Auxologico Italiano; ${ }^{2}$ Division of Rheumatology, Asst Pini-CTO;

${ }^{3}$ Department of Clinical Sciences and Community Health, Università degli Studi di Milano, Milan, Italy; ${ }^{4}$ Research and Development, Inova Diagnostics, San Diego, USA; ${ }^{5}$ Department of Clinical and Experimental Medicine, Rheumatology Section, Second University of Naples, Naples; ${ }^{6}$ Department of Rheumatology, University of L'aquila, School of Medicine, L'Aquila, Italy

Background: Systemic sclerosis (SSc) is a chronic autoimmune disease charac terised by vascular changes and progressive fibrosis of skin and various internal organs. In SSc a variety of autoantibodies have been detected which are usefu for the diagnosis and management of the disease. Some of these autoantibodies are well-established tools strongly associated with SSc (e.g. anti-centromere, anti-topoisomerase I, anti-RNA polymerase III). Other autoantibodies are less frequent and/or less-specific for SSc even if potentially useful to better assess disease subsets and prognosis.

Objectives: Our goal was to assess the frequency of SSc-related autoantibodies detected using a novel technology as well as to study the associations between these antibodies and clinical features in an Italian SSc cross sectional cohort.

Methods: Serum samples from 218 consecutive patients with SSc collected at three Italian sites were tested for a variety of autoantibodies (see table 1) using a novel fully automated system utilising bead-based immunoassays (research use only, Inova Diagnostics, San Diego, CA). The Italian cohort included: women 200 (92\%), limited cutaneous SSc (Ic-SSc) $166(76 \%)$, patients with history of digital ulcers $91(42 \%)$, calcinosis $46(21 \%)$, lung fibrosis $84(39 \%)$, heart involvement 38 $(17.4 \%)$, pulmonary arterial hypertension $20(9 \%)$, and esophageal involvement $138(63.3 \%)$.

Results: The prevalence of antibodies is summarised in the table 1 below. Of note, anti-BICD2, anti-CENP-B, and anti-nucleosome antibodies were significantly associated with Ic-SSc subtype $(p=0.0237, p<0.0001, p=0.0096$, respectively), while anti-Ro60, anti-SSB, anti-Scl-70, and anti-DFS70 antibodies were significantly associated with the diffuse cutaneous SSc (dc-SSc) subtype $(p=0.0102, p<0.0001, p<0.0001$, and $p=0.0032$, respectively). When analysing all antibodies with multivariate analysis, SSc patients showed significant clustering based on antibody profile and clinical phenotype.

\begin{tabular}{|c|c|c|c|c|}
\hline Antibody & Total SSC No. (\%Pos, 95\% Cl) & dc.-SSc No. (\%Pos, 95\% Cl) & IC.-55c No. (\%)Pos, 95\% Cl) & $\begin{array}{c}\text { p-value } \\
\text { (dc.SSc vs. Ic.SSc) }\end{array}$ \\
\hline dSDNA & $10(4.6 \%, 95 \% \mathrm{Cl} 2.5-8.2)$ & $2(3.8 \%, 1.1-1.30 \%)$ & $8(4.8 \%, 2.5-9.2 \%)$ & N.S. \\
\hline RNP & $11(5.0 \%, 95 \% \mathrm{Cl} 2.8-8.8)$ & $3(5.8 \%, 2.0-15.6 \%)$ & $8(4.8 \%, 2.5-9.2 \%)$ & N.S. \\
\hline Ro52 & $32(14.7 \%, 95 \%$ Cl 10.6-20.0) & $10(19.2 \%, 10.8-31.9 \%)$ & $22(13.3 \%, 8.9-19.3 \%)$ & N.S. \\
\hline Ro60 & $15(6.9,95 \%$ C $14.2-11.0)$ & $8(15.4 \%, 8.0-27.5 \%)$ & $7(4.2 \%, 2.1-8.4 \%)$ & 0.0102 \\
\hline SS-B & $7(3.2 \%, 95 \%$ C1 1.6-6.5) & $7(13.5 \%, 6.7-25.3 \%)$ & $0(0.0 \%, 0.0-2.3 \%)$ & $<0.0001$ \\
\hline $\mathrm{Scl}-70$ & $59(27.1 \%, 95 \%$ C1 121.6-33.3) & $39(75.0 \%, 61.8-84.8 \%)$ & $20(12.0 \%, 7.9 \cdot 17.9 \%)$ & $<0.0001$ \\
\hline DF570 & $6(2.8 \%, 95 \%$ Cl 1.3 .5 .5$)$ & $5(9.6 \%, 4.2 \cdot 20.6 \%)$ & $1(0.6 \%, 0.1-3.3 \%)$ & 0.0032 \\
\hline Jo-1 & $2(0.9 \%, 95 \%$ C $10.3-3.3 .3)$ & $1(1.9 \%, 0.3 \cdot 10.1 \%)$ & $1(0.6 \%, 0.1-3.3 \%)$ & N.S. \\
\hline CENP-B & $111(50.9 \%, 95 \%$ C $144.3-57.5)$ & $5(9.6 \%, 4.2-20.6 \%)$ & $106(63.9 \%, 56.3-70.8 \%)$ & $<0.0001$ \\
\hline Nucleosome & $38(17.4 \%, 95 \%$ C $113.0-23.0)$ & $15(28.8 \%, 18.3-42.3 \%)$ & $23(133.9 \%, 9.4-19.9 \%)$ & 0.0096 \\
\hline Ribo-p & $1(0.5 \%, 95 \%$ C10.1-2.6) & $1(1.9 \%, 0.3-10.1 \%)$ & $0(0.0 \%, 0.0-2.3 \%)$ & N.S. \\
\hline $\mathrm{sm}$ & $0(0.0 \%, 95 \%$ C1 $10.0-1.7)$ & $0(0.0 \%, 0.0-6.9 \%)$ & $0(0.0 \%, 0.0-2.3 \%)$ & N.S. \\
\hline Ku & $6(2.8 \%, 95 \% \mathrm{Cl} 1.3-5.9)$ & $2(3.8 \%, 2.2-13.0 \%)$ & $4(2.4 \%, 0.9-6.0 \%)$ & N.S. \\
\hline PCNA & $1(0.5 \%, 95 \% \mathrm{Cl} 0.1-2.6)$ & $0(0.0 \%, 0.0-6.9 \%)$ & $1(0.6 \%, 0.1-3.3 \%)$ & N.S. \\
\hline RNA POI III & $4(1.8 \%, 95 \% \mathrm{Cl} 0.7-4.6)$ & $2(3.8 \%, 2.2 \cdot-13.0 \%)$ & $2(1.2 \%, 0.3-4.3 \%)$ & N.S \\
\hline Rpp25 & $2(0.9 \%, 95 \% \mathrm{Cl} 0.3-3.3)$ & $0(0.0 \%, 0.0-6.9 \%)$ & $2(1.2 \%, 0.3-4.3 \%)$ & N.S \\
\hline Rpp 38 & $3(1.4 \%, 95 \%(10.5-4.0)$ & $0(0.0 \%, 0.0-6.9 \%)$ & $3(1.8 \%, 0.6-5.2 \%)$ & N.S \\
\hline PmScl & $10(4.6 \%, 95 \% \mathrm{Cl} 2.5-8.2)$ & $1(1.9 \%, 0.3-10.1 \%)$ & $9(5.4 \%, 2.9-10.0 \%)$ & N.S. \\
\hline $\mathrm{BICD} 2$ & $51(23.4 \%, 95 \%$ C1 18.3-29.4) & $6(11.5 \%, 5.4-23.0 \%)$ & $45(27.1 \%, 20.9-34.3 \%)$ & 0.0237 \\
\hline Мाтз & $18(8.3 \%, 95 \%$ C1 $5.3-12.7)$ & $2(3.8 \%, 1.1 \cdot 13.0 \%)$ & $16(9.6 \%, 6.0-15.1 \%)$ & N.S. \\
\hline$\angle K M-1$ & $0(0.0 \%, 95 \% \mathrm{C}(0.0-1.7)$ & $0(0.0 \%, 0.0-6.9 \%)$ & $0(0.0 \%, 0.0-2.3 \%)$ & N.S. \\
\hline SLA & $1(0.5 \%, 95 \%$ C $10.1-2.6)$ & $0(0.0 \%, 0.0-6.9 \%)$ & $1(0.6 \%, 0.1-3.3 \%)$ & N.S. \\
\hline sp100 & $2(0.9 \%, 95 \%$ C $10.3-3.3 .3)$ & $1(1.9 \%, 0.3-10.1 \%)$ & $1(0.6 \%, 0.1 \cdot 3.3 \%)$ & N.S. \\
\hline gp 210 & $2(0.9 \%, 95 \% \mathrm{Cl} 0.3-3.3)$ & $1(1.9 \%, 0.3-10.1 \%)$ & $1(0.6 \%, 0.1-3.3 \%)$ & N.S. \\
\hline $\mathrm{VCP}$ & $0(0.0 \%, 95 \%$ C1 $10.0-1.7)$ & $0(0.0 \%, 0.0-6.9 \%)$ & $0(0.0 \%, 0.0-2.3 \%)$ & N.S. \\
\hline LC-1 & $1(0.5 \%, 95 \% \mathrm{Cl} 0.1-2.6)$ & $0(0.0 \%, 0.0-6.9 \%)$ & $1(0.6 \%, 0.1-3.3 \%)$ & N.S. \\
\hline HK-1 & $5(2.3 \%, 95 \%$ C $11.0-5.3)$ & $2(3.8 \%, 2.2 \cdot-13.0 \%)$ & $3(1.8 \%, 0.6-5.2 \%)$ & N.S \\
\hline Kelch & $2(0.9 \%, 95 \%$ C $10.3-3-3.3)$ & $0(0.0 \%, 0.0-6.9 \%)$ & $2(1.2 \%, 0.3-4.3 \%)$ & N.S \\
\hline
\end{tabular}

N.S. = not significant

Conclusions: Autoantibodies identified via the novel system in this SSc cohort were found in the expected frequencies and also correlated to clinical features of the patients. The multiparameter approach combined with cluster analysis holds promise for a molecular and more precise stratification of SSc subsets.

Disclosure of Interest: None declared

DOI: 10.1136/annrheumdis-2018-eular.5688 